\title{
Modeling of SEDs for substars with disks that have different geometrical and physical parameters
}

\author{
Olga Zakhozhay ${ }^{1}$ \\ ${ }^{1}$ Main Astronomical Observatory National Academy of Sciences of Ukraine, \\ 27 Akademika Zabolotnoho St. 03680 Kyiv, Ukraine \\ email: zkholga@mail.ru
}

\begin{abstract}
The algorithm of spectral energy distribution (SED) calculations for protoplanetary disks and central objects is created. The results of SEDs calculations for substars with protoplanetary disks that have a different ages and inclinational angles are discussed.
\end{abstract}

Keywords. circumstellar matter, stars: low-mass, brown dwarfs, planetary systems: protoplanetary disks

\section{Introduction}

At the beginning of this century a physical model for substellar (brown dwarf) evolution was created by research group from Astronomical Institution of V.N. Karazin Kharkiv National University (Pisarenko et al. 2007). These models describe the evolution of the substars with masses $0.01-0.08$ solar masses and ages within the range of $1 M y r-10 G y r$.

Based on these investigations the models of disks that would surround such substars were simulated and SEDs were calculated. With this algorithm it is possible to calculate SEDs for substar (or star) with disk that inclined on any angle with taking into account all geometrical particularities that system can have.

The results for the following cases have being received:

- substar masses within the range of $0.01-0.08 M_{\text {sol }}$;

- protoplanetary disks with different inclination angles $\left(0^{\circ}-80^{\circ}\right)$;

- systems are in the age interval from 1 to 30 Myr;

- substars and protoplanetary disks are radiate like black body based on the Chiang \& Goldreich (1997) model;

- distance from Sun to substar equals to $10 p c$;

- disk's inner radius equals to central object radius and sublimation radius in the age of $1 M y r$.

\section{Results}

1120 SEDs for systems with all variety of above-mentioned parameters have being received. In this work a case of flat disk without inner hole that surround a substar with mass $0.08 M_{\text {sol }}$ (as an example) is discussed. On Fig. 1 the evolution of SED's shape and intensity with the age (left panel) and inclination angle (right panel) is shown.

Calculational models were compared with observational data that were received by Scholz et al. (2007). On Fig. 2 SED's and observational data for two objects usd 15556 and usd155601 are shown. SED's were calculated using parameters that were derived by Scholz et al. (2007): for usd155556 - substellar mass $M_{s s}=0.075 M_{\text {sol }}$, substellar 

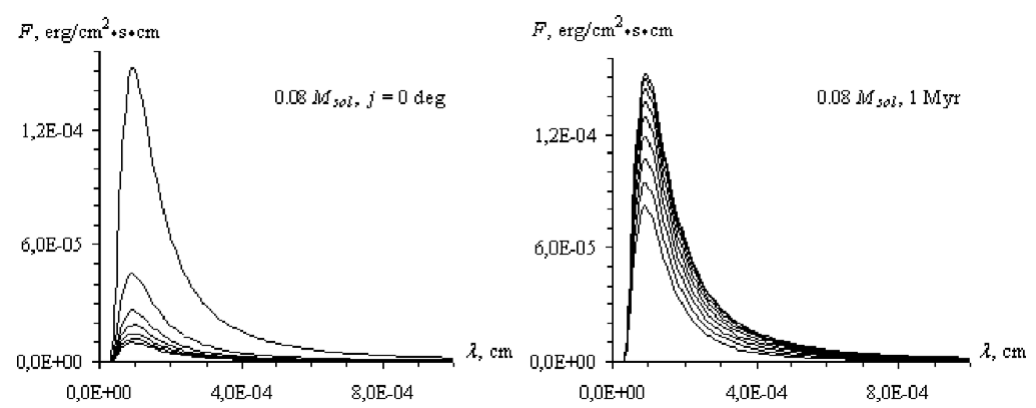

Figure 1. SEDs for systems with substar $\left(0.08 M_{s o l}\right)$ and flat disk without inner hole: left panel- different curves show different system ages (from top to bottom): 1 Myr, 5Myr, 10Myr, 15Myr, 20Myr, 25Myr and 30Myr; right panel - different curves show different system inclinations (from top to bottom): $0^{\circ}, 10^{\circ}, 20^{\circ}, 30^{\circ}, 40^{\circ}, 50^{\circ}, 60^{\circ}, 70^{\circ}$ and $80^{\circ}$.
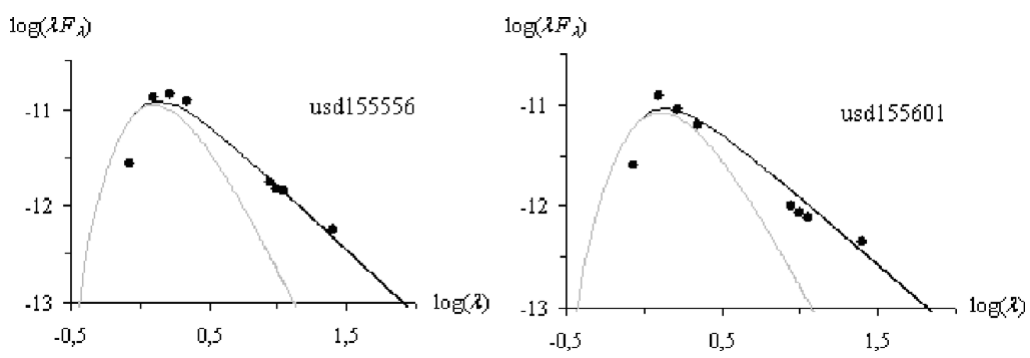

Figure 2. Comparison of observational data (points) with modeled SEDs (solid black line), substellar black body SED is shown with solid grey line

temperature $T_{s s}=2900 \mathrm{~K}$, substellar radius $R_{s s}=0.4 R_{s o l}$; for usd155601- $M_{s s}=$ $0.075 M_{s o l}, T_{s s}=2900 K, R_{s s}=0.35 R_{\text {sol }}$; disks of both objects have inner radius $R_{i n}=$ $R_{s s}$, outer radius $R_{o u t}=100 a u$ and inclination angle $20^{\circ}$.

\section{Conclusions}

- The algorithm of SEDs calculations for substars with disks with and without inner hols, different ages, different geometry and inclination angle has been developed.

- 1120 SEDs for systems with substars within the masses of $(0.01-0.08) M_{\text {sol }}$ at the age $1-30$ Myr and flat or flared disks that inclined on different angles have been calculated.

- SEDs that were calculated using our model are in agreement with SEDs that were calculated by Scholz et al. (2007).

\section{Acknowledgments}

Author want to acknowledge International Astronomical Union and Astronomical Institute of Ruhr University (Germany) for the financial support that gives an opportunity to participate in IAU Symposium 276.

\section{References}

Pisarenko, A. I., Yatsenko, A. A., \& Zakhozhay, V. A. 2007, Astron. Rep., 51, 605

Chiang, E. I. \& Goldreich, P. 1997, ApJ, 490, 368

Scholz, A., Jayawardhana, R., Wood, K., et al. 2007, ApJ, 660, 1517 